\title{
A RING WHICH IS A DOMAIN LOCALLY BUT NOT GLOBALLY
}

\author{
MOSHE ROITMAN
}

\begin{abstract}
We present here a connected commutative ring $R$ which is not a domain, but $R_{P}$ is a domain for any prime ideal $P$ of $R$.
\end{abstract}

It is well known that if $R$ is a connected (= without nontrivial idempotents) commutative noetherian ring such that $R_{M}$ is a domain for any maximal ideal $M$ of $R$, then $R$ itself is a domain $[\mathbf{1}, 13.14]$. We show here that this assertion does not remain true if we drop the noetherian assumption on $R$, thus answering the question in $[\mathbf{1}, 13]$ : We define inductively a sequence of rings $R_{n}(n \geq 0)$ :

Let $R_{0}=F[X, Y] /(X Y)$, where $F$ is a field of characteristic 2 . In case $n>0$, adjoin to $R_{n-1}$ indeterminates $X_{a, b}$ for any $a, b$ in $R_{n-1}$ such that $a b=0$. Let $I_{n}$ be the ideal in the polynomial ring $R_{n-1}\left[X_{a, b}\right]_{\left\{a, b \in R_{n-1}: a b=0\right\}}$ generated by $\left\{a X_{a, b}, b\left(1-X_{a, b}\right): a, b \in R_{n-1}, a b=0\right\}$. Let $R_{n}=R_{n-1}\left[X_{a, b}\right] / I_{n}$. We have canonical homomorphisms $\varphi_{n}: R_{n} \rightarrow R_{n+1}$. Let $R=\lim$ ind $R_{n}$.

Proposition. $R \neq 0$ is connected, $R$ has zero divisors, but $R_{P}$ is a domain for any prime ideal $P$.

To prove the Proposition we need some lemmas. We denote $T$, a commutative ring with $1 \neq 0 ; a, b$ are elements of $T$ such that $a b=0 ; I$ is the ideal $(a X, b(1-X))$ in $T[X] ; T^{\prime}=T[X] / I$ and $\varphi: T[X] \rightarrow T^{\prime}$ is the natural homomorphism.

LEMMA 1. We have $t^{2}=0$ for any $t \in T \cap I$.

Proof. Let $t \in T \cap I, t=f a X+g b(1-X)$, where $f, g$ are in $T[X]$. Then $t=g(0) b=f(1) a$, so $t^{2}=g(0) f(1) a b=0$.

LEMMA 2. If $t \in T$ is not nil in $T$, then $\varphi(t)$ is not nil in $T^{\prime}$.

PROOF. If $t \in T$ and $\varphi(t)$ is nil in $T^{\prime},(\varphi(t))^{m}=0$, then $t^{m} \in T \cap \operatorname{ker} \varphi$, so by Lemma $1, t^{m}$ is nil, $t$ is nil.

LEMMA 3. If $T$ is connected, char $T=2$, then $T^{\prime}$ is also connected.

Proof. Let $e(X)=e_{0}+e_{1} X+\cdots+e_{k} X^{k} \in T[X], \varphi(e)$ idempotent in $T^{\prime}$. As $\left(e_{0}+e_{1} X+\cdots+e_{k} X^{k}\right)^{2}-\left(e_{0}+\cdots+e_{k} X^{k}\right) \in(a X, b(1-X))$, we obtain in $T: e_{i} \in \sqrt{(a, b)}$ for $1 \leq i \leq k$. Indeed, assume $(a, b) \neq(1), \bar{T}=T /(a, b)$ and for $t \in T$, let $\bar{t}$ be the canonical image of $t$ in $T$. Then $\bar{e}_{0}+\cdots+\bar{e}_{k} X^{k}$ is an idempotent in $\bar{T}[X]$, so $\bar{e}_{i}$ is nil for $1 \leq i \leq k$, that is $e_{i} \in \sqrt{(a, b)}$ for $1 \leq i \leq k$.

If $r$ is sufficiently big, then $e_{i}^{2^{r}} \in(a, b)$ for $1 \leq i \leq k$, so there exists $t$ in $T$ such that

$$
\left(e_{0}+\cdots+e_{k} X^{k}\right)^{2^{r}}=e_{0}^{2^{r}}+\cdots+e_{k}^{2^{r}} X^{2^{r} k} \equiv t \quad(\bmod (a X, b(1-X))) .
$$

Received by the editors January 26, 1984 and, in revised form, May 18, 1984.

1980 Mathematics Subject Classification. Primary 13G05, 13A17.

(C) 1985 American Mathematical Society $0002-9939 / 85 \$ 1.00+\$ .25$ per page 
It follows $t^{2} \equiv t(\bmod I)$, and so by Lemma 1 , we have $\left(t^{2}-t\right)^{2}=t^{4}-t^{2}=0$. Therefore $t^{2}=0$ or $t^{2}=1$ and so $e^{2^{r+1}} \equiv 0(\bmod I)$ or $e^{2^{r+1}} \equiv 1(\bmod I)$. As $e^{2^{r+1}} \equiv e(\bmod I)$, the lemma is proved.

Proof OF THE Proposition. Let $\psi: R_{0} \rightarrow R$ be the canonical homomorphism. From Lemma 2 it follows that $\psi$ is a monomorphism, so $R \neq 0$ has zero divisors. From Lemma 3 it follows that $R$ is connected. By construction, if $x y=0$ in $R$, then $\operatorname{Ann}(x)+\operatorname{Ann}(y)=R$, so $R_{P}$ is a domain for any prime ideal $P$ of $R$.

\section{REFERENCES}

1. A. V. Geramita and C. Small, Introduction to homological methods in commutative rings, Queen's Papers in Pure and Applied Mathematics, No. 43, Queen's Univ., Kingston, Ontario, 1976.

Department of Mathematics, University of haifa, Mount Carmel, haifa 31999, ISRAEL 